\title{
T U Secondary causes for osteoporosis significantly contribute to fracture risk in patients with osteopenia and a recent fracture
}

Frank Malgo ${ }^{1,4}$, Natasha M. Appelman-Dijkstra ${ }^{1,4}$, Frank Termaat ${ }^{2}$, Huub J.L. van der Heide ${ }^{3}$, Inger B. Schipper ${ }^{2}$, Neveen A.T. Hamdy ${ }^{1,4}$

Departments of Endocrinology and Metabolic Diseases ${ }^{1}$, Traumatology ${ }^{2}$, Orthopedic Surgery ${ }^{3}$ and Center for Bone Quality ${ }^{4}$, Leiden University Medical Center, Leiden, The Netherlands

\section{Introduction}

- Secondary causes for osteoporosis are prevalent in up to $60 \%$ of patients with osteoporosis and/or a fracture.

- Fractures are prevalent in patients with osteopenia.

- Data on secondary causes for osteoporosis are scarce in patients with osteopenia.

\section{Objective}

To evaluate whether secondary causes for osteoporosis are prevalent, and may contribute to fracture risk, in patients aged $\geq 50$ years with osteopenia and a recent fracture.

\section{Patients and methods}

606 consecutive men and women aged $\geq 50$ years presenting with a recent fracture were included in the study.

Patients $\geq 50$ years old and recent fracture

Bone Mineral Density (DXA) Screening laboratory investigations

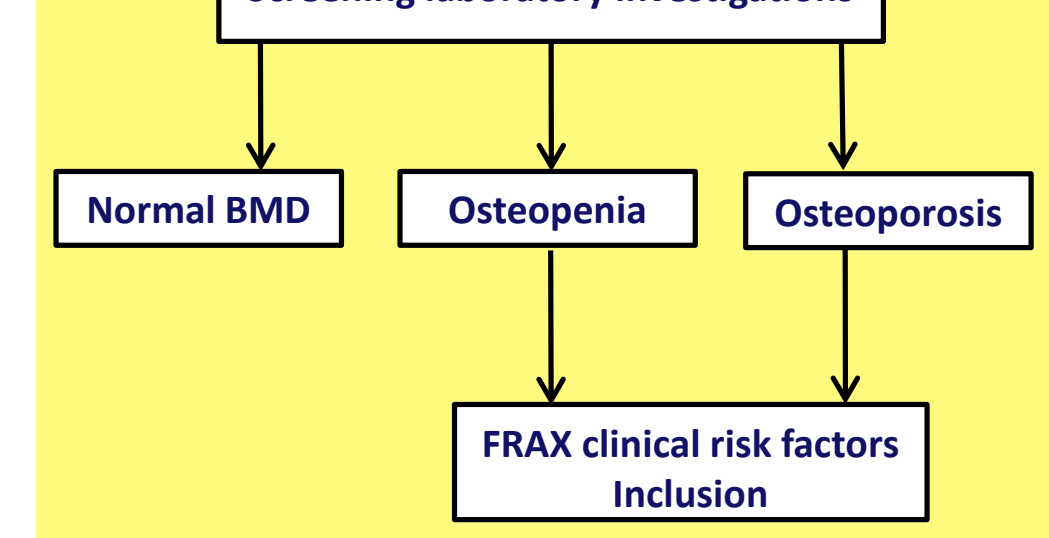

345 (57\%) patients with osteopenia were used as index group $174(29 \%)$ patients with osteoporosis were used as controls

\section{Results}

Demographic characteristics of $\mathbf{3 4 5}$ patients with osteopenia and a recent fracture

Age (years)

$66,1 \pm 10,0$

Male/female

$99 / 246$

$\mathrm{BMI}\left(\mathrm{kg} / \mathrm{m}^{2}\right)$

$26,8 \pm 4,1$

25-OH vitamin D (nmol/L)

Previous fracture VF/NVF (\%)

$56,2 \pm 27,1$

FRAX score major OP fracture

$170(49)$

FRAX score hip fracture

$10,0 \pm 5,7$

$3,1 \pm 3,9$

Correlation between number of secondary causes for osteoporosis and BMD at the femoral neck

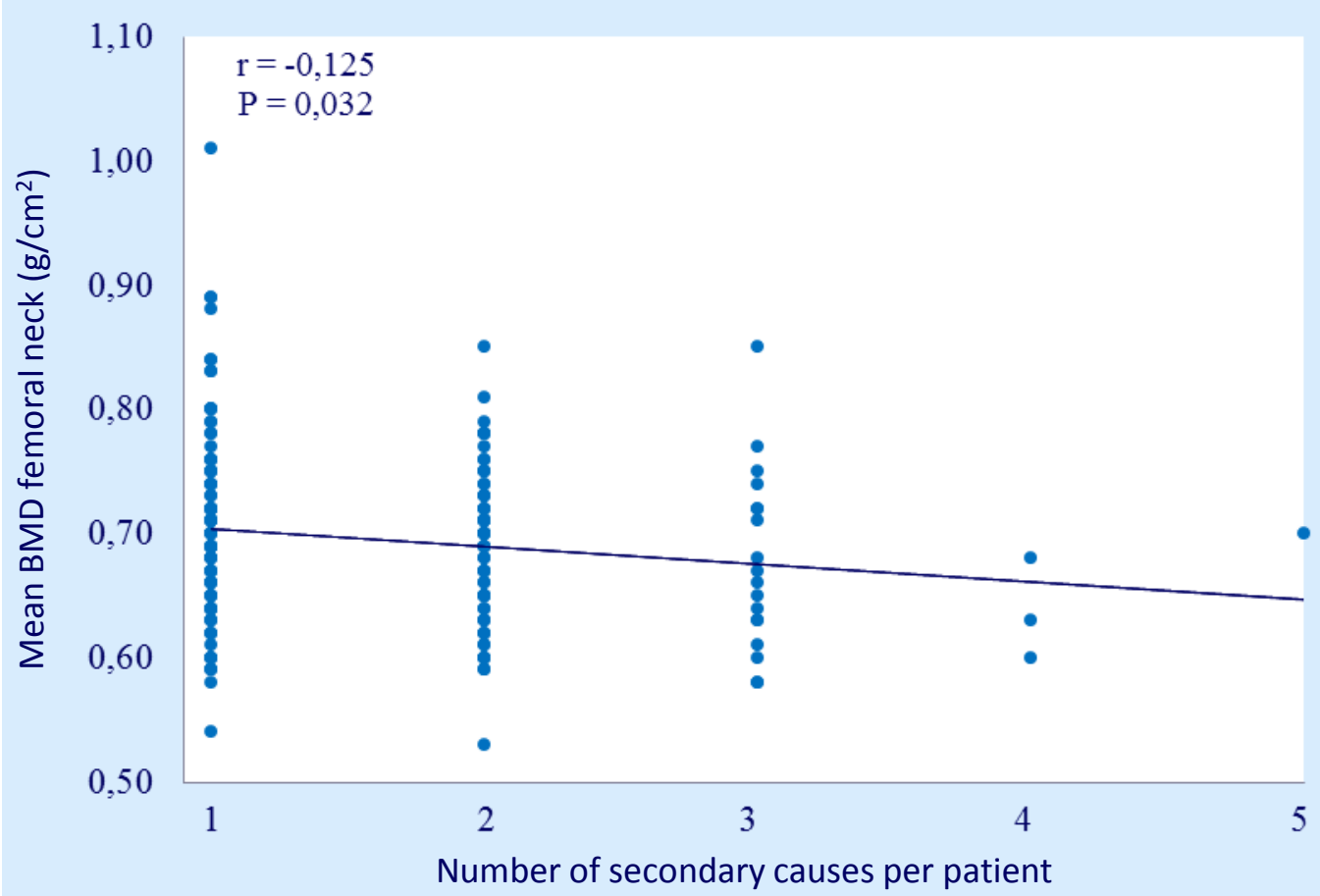

Prevalence of secondary causes by FRAX
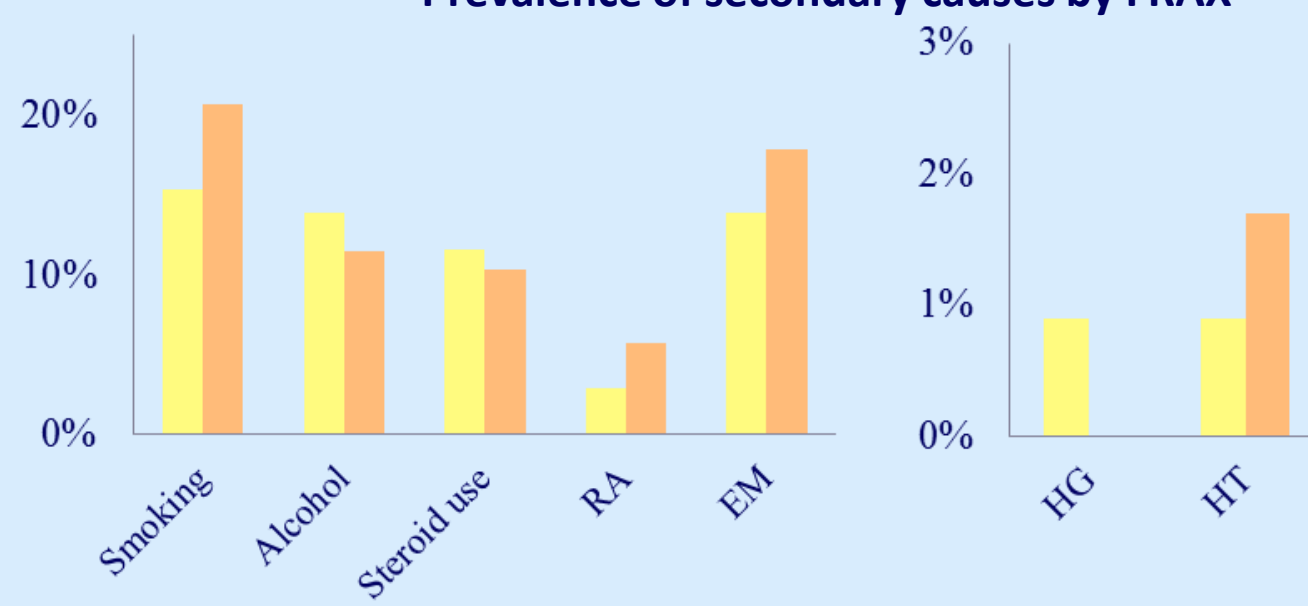

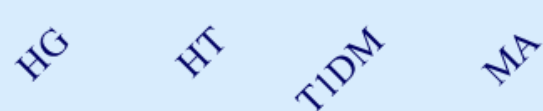

Prevalence of secondary causes by screening laboratory tests

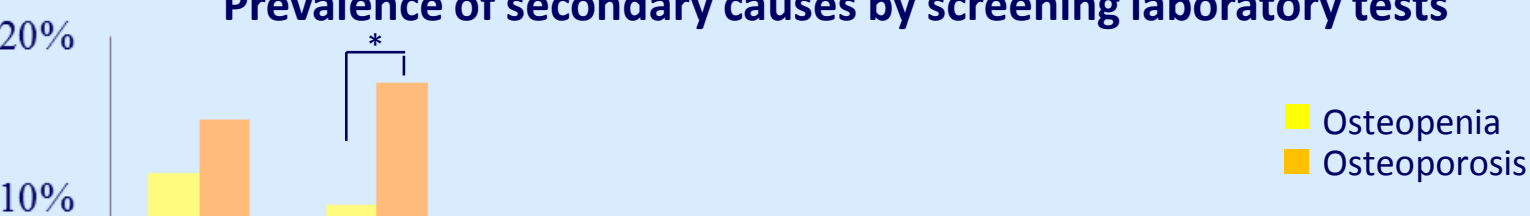

$10 \%$

$0 \%$

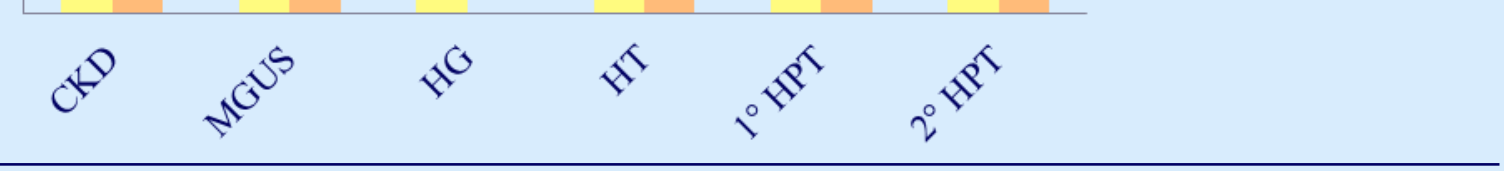

RA rheumatoid arthritis; EM early menopause; HG hypogonadism; HT hyperthyroidism; T1DM type 1 diabetes mellitus; MA malabsorption; CKD chronic kidney disease; MGUS monoclonal gammopathy of undetermined significance; HPT hyperparathyroidism; *P $<0,05$

- $76 \%$ of patients with osteopenia had $\geq 1$ secondary cause for osteoporosis vs. $\mathbf{8 1 \%}$ of patients with osteoporosis

- $47 \%$ of patients with osteopenia had $25-\mathrm{OH}$ vitamin D insufficiency vs. $44 \%$ of patients with osteoporosis

$32 \%$ of causes amenable to lifestyle changes; $39 \%$ were treatable

\section{Conclusion}

1. Secondary causes for osteoporosis are highly prevalent in patients with osteopenia and a recent fracture, $48 \%$ of which were identifiable by FRAX and $54 \%$ by laboratory investigations.

2. Secondary causes for osteoporosis may contribute to fracture risk not only by decreasing bone mass but also by altering bone quality. 